\title{
La compleja identidad personal
}

\section{The Complex Personal Identity}

\author{
Luis Álvarez-Munárriz \\ Área de Antropología Social. Facultad de Filosofía. \\ Universidad de Murcia
}

\section{RESUMEN}

En este trabajo se pretende poner de manifiesto la relevancia teórica que tiene la identidad personal y su importancia en la vida de las personas. Se exponen los principales marcos teóricos que se han usado para entenderla. Se explica cómo en el campo de la antropología social la identidad personal ha sido diluida en la estructura sociocultural. Para recuperarla se propone y fundamenta una visión ecosistémica de la categoría de persona.

Palabras clave: Identidad, Modelos de identidad, Giro ontológico, Persona, Ontología ecosistémica, Conciencia.

\section{SUMMARY}

This paper seeks to show the theoretical relevance of the personal identity and its value for the life of every person. An explanation is given of the theoretical framework that has been used to understand both the personal identity and how, in the Social Anthropology field, it has been diluted within the social structure. An eco-systemic vision of the person category is proposed and founded to recover the lost personal identity.

Key words: Identity, Models of Identity, Ontological Turn, Person, Eco-systemic Ontology, Consciousness.

La identidad se puede describir como la conciencia y la asunción de unos modos de ser, pensar y actuar que dotan de significado y sentido a la vida de una persona ${ }^{1}$. Este rasgo del ser humano no se sustenta en una esencia inmutable, sino que remite a una estructura entitativa estable, dinámica y creativa, ya que se construye y se vive no en la clausura y el aislamiento sino en la interacción con los miembros del grupo al que pertenece y dentro de un medio físico concreto. Cada persona es un ser completo en sí

\footnotetext{
${ }^{1}$ El soporte etnográfico en el que se apoya esta contribución se puede encontrar en el libro de Álvarez Munárriz, y otros (2011: 154-172).
} 
mismo pero en íntima relación con los demás. Ser persona exige la presencia y la relación con los otros. Las maneras de estructurar estas formas de relación siempre están moduladas por la cultura de cada sociedad, pero en manera alguna podemos ignorar o excluir el sentimiento de identidad personal puesto que es un atributo esencial en la vida de cualquier individuo. En suma: la identidad personal es substantiva y al mismo tiempo relacional, es decir, compleja: "Our identity, by definition, is not what makes us the same as others but what makes us unique. Identity is a complex and contextually sensitive combination of elements" (Council of Europe 2008: 5).

\section{MODELOS DE IDENTIDAD PERSONAL}

Esta compleja identidad de las personas no se puede desvelar y esclarecer con los métodos tradicionales que hasta ahora han venido usando las ciencias positivas para explicar la estructura y el funcionamiento de la realidad. La identidad personal no es un objeto que se pueda delimitar, fijar y aislar para poder analizar o realizar experimentos. El enfoque naturalista no tiene en cuenta el mundo vital y la relaciones de comunicación que en él establecen los seres humanos y que de ninguna manera se pueden reducir al mero cálculo y la formalización. Es el ámbito sociocultural en el que los seres humanos, tanto a nivel individual como colectivo, ejercen, invocan o reclaman su propia identidad. Este campo remite a un conjunto de procesos vitales donde tan esencial es la persona como el contexto en el que ésta se desenvuelve. Es en este todo complejo donde inicia y concluye su trabajo de campo el antropólogo. En este escenario observa y participa con las personas que lo conforman y en el que cada una de ellas representa múltiples papeles. No es de extrañar, por tanto, que sean muchos los antropólogos que afirman que actuamos como agentes múltiples, que representamos muchos papeles. $\mathrm{Y}$ en este supuesto se han basado para entender la identidad individual como la combinación de cada una de las diversas identidades sociales que de forma simultánea o alternativa puede asumir una persona (Olmo 1994: 81; González García 2001: 59).

Un detenido análisis de los datos que aporta el trabajo de campo obligaría a concluir que entre esas dimensiones de la identidad no existe ni separación ni jerarquía, sino que son como caras de una misma moneda. La identidad social se refiere a un sentimiento de similitud con otros; la identidad personal se refiere a un sentimiento de diferencia en relación con esos mismos otros (Deschamps y Devos 1996: 42). Aunque las separemos en nuestras investigaciones o exposiciones, se debe subrayar que son expresiones de un todo complejo pero unitario, partes inseparables que se 
conjugan y diluyen en el sistema dinámico y relacional que cada uno de nosotros somos. Pero es precisamente la dificultad que entraña explicar estas dimensiones de la identidad que ejerce el individuo en la vida diaria — "Un hombre son muchos hombres" nos dirá el poeta- lo que justifica el estudio pormenorizado de la identidad personal. El antropólogo debe abordar esta dimensión de la identidad porque sustenta modos de ser y actuar que son determinantes en el discurrir de la vida diaria de cualquier persona. En efecto, la concepción que se tiene de uno mismo y el modo cómo se vive un papel importante en la vida de la gente en la medida en que es fuente de sentido y experiencia para sus vidas: el amor, la amistad, la responsabilidad laboral, los derechos y obligaciones, la forma en la que nos vemos unos a otros, el reconocimiento, la sanción, etc. Las creencias que los seres humanos tienen acerca de ellos mismos constituyen una fuerza poderosa en su vida individual y colectiva. La manera cómo nos percibimos a nosotros mismos constituye una especie de faro que, tanto a nivel consciente como inconsciente, orienta e influye en nuestras preferencias y decisiones.

Todos los científicos sociales que abordan el tema de la identidad coinciden en la necesidad de construir, para poderlo usar en sus investigaciones, un modelo teórico en el que se consiga una síntesis de la dimensión individual y social de la identidad, una visión unitaria de las que con frecuencia se han designado como la dimensión intrapersonal y la extrapersonal, una conjunción de las estructuras endógenas y exógenas, una integración teórica del paradigma normativo e interpretativo. Necesitamos un modelo que distinga epistemológicamente entre niveles de explicación referidos al individuo y niveles relativos a lo social sin que se aplasten el uno al otro o se diluya alguno de ellos. El reto consiste en complementar ambos aspectos dentro un modelo en el cual tanto la identidad de las personas como su presencia en las redes sociales estén acopladas y garantizadas (Lisón Tolosana 2010: 168; Sánchez de la Yncera 2008: 105; Kreiner y otros 2006: 1037; Bertaux 2005: 20). Pues bien, si rastreamos la historia del pensamiento podemos constatar que este objetivo se ha pretendido alcanzar con tres modelos diferentes:

a) Ontológico. En el campo de la filosofía clásica, desde un enfoque estrictamente ontológico: una sustancia coincidente y cerrada en sí misma. En este hecho se fundamenta el sentimiento unificado que todos tenemos de ser un solo ente y diferente de los demás. La pregunta quién soy yo se responde y explica con la categoría de persona. Con palabras de Boecio: "sustancia individual de naturaleza racional" y que un filósofo español, muchos siglos después, condensó en la siguiente expresión: "Realidad formalmente 
suya" (Zubiri 1986: 110). Es el rasgo que hace que un ser humano sea un ser uno y único y que posea, por tanto, una identidad propia y específica. Según este enfoque teórico todo ser humano posee una estructura ontológica estable que constituye la base que canaliza las posibles transformaciones que a lo largo del tiempo soportan los seres humanos de cualquier época o lugar.

b) Psicosocial. Dos líneas de pensamiento confluyen en la configuración de este modelo teórico. De una parte la que surge de las aceradas críticas de Locke a la visión sustancial del ser humano. La recoge, y es clave, el rechazo de W. James a cualquier visión sustancial de la conciencia, la desarrolla G. H. Mead con su concepto del self ( $I$ y me), y culmina con las aportaciones de Erickson sobre el concepto de identidad: sentimiento subjetivo de permanencia personal y de continuidad temporal garantizado por la memoria y fundado en el reconocimiento que los demás hacen de nuestra unidad y persistencia personal. De otra parte, la línea que proviene de Marx, quien condensa su teoría en el siguiente axioma: es su ser social lo que determina la conciencia de cada hombre. La sociedad fabrica la conciencia del individuo y en consecuencia la identidad de las personas. Son dos líneas paralelas que están comenzando a converger. Los representantes de las ciencias empíricas afirman: somos nuestro cerebro. Y los representantes de las ciencias humanas y sociales responden: es esa una condición necesaria pero no suficiente, pues se requiere la impronta social para su configuración. Pero prescindiendo de los enfrentamientos y rechazos disciplinares, todos empiezan a coincidir ya en esta tesis: el substrato de la conciencia o memoria humana es, por esencia, social.

c) Ecosistémico. Más recientemente ha surgido un modelo de identidad que hunde sus raíces en la ideas de G. H. Mead sobre organismo, entorno y emergencia, y que culmina en el presente en las denominadas ciencias de la complejidad. En ellas se ve al sujeto como un sistema unitario que se autoorganiza dentro de un medio complejo y que tiene como resultado su propia individualización. Todas las relaciones de producción están coordinadas en un sistema que mantiene íntegra su identidad y autonomía a pesar de las perturbaciones a las que constantemente está sometido. En esta orientación holística es clave la noción de "sistema complejo adaptativo" que posee la capacidad de modular las constricciones internas y externas que soporta a lo largo del tiempo. La identidad de la persona se fundamenta en el concepto de sistema dinámico entendido como un proceso de co-emergencia de interioridad y exterioridad en el que se desarrollan niveles más complejos e integrados de auto-identidad.

La exposición sintética de estos tres marcos teóricos no debe ser vista como un ejercicio de erudición. Se debe entender como una llamada de 
atención para que se comprenda y reconozca la importancia, actualidad y necesidad de complementar estos tres enfoques, recuperando aquellos puntos fuertes que permiten sugerir planos secantes de complementariedad. Criticar y rechazar pero también aceptar, fusionar, actualizar y mantener las contribuciones de cada uno de estos tres marcos es la única manera de poder evitar las tautologías y las paradojas que entraña el intento de avanzar en la comprensión de la compleja identidad personal. Y es que, más allá de cuestiones terminológicas y enfrentamientos académicos, es necesario subrayar que estos tres enfoques son compatibles y además necesarios para poder acercarnos a una concepción más sólida y fértil de la identidad personal. Absolutamente necesarios, porque el modelo imperante en los usos explicativos de las ciencias humanas y sociales acerca de la identidad personal y social es difuso y escasamente fértil. Esas carencias se pueden constatar reflexionando sobre un intento de síntesis reciente que se condensa en este texto:

La concepción del sujeto humano que creo que permite formular un concepto razonable de autonomía personal en la actualidad se encuentra dispuesta en una determinada tradición del intersubjetivismo; en ella se conjugan el conocimiento de la constitución inter subjetiva de la identidad del yo y el conocimiento de que a la experiencia consciente del hombre siempre se le escapa una parte de las fuerzas y los motivos que constituyen su potencial impulsor psíquico (Honneth 2010: 282).

En este modelo la mente humana parece concebirse como una hoja en blanco en la que la sociedad va escribiendo su particular versión de sus propios impulsos, deseos y costumbres a través de un proceso de enculturación. La actividad consciente que podemos observar en el comportamiento social de cualquier sujeto se remonta y tiene como base la dimensión inconsciente en la que se sedimentan las estructuras de la cultura de la sociedad. Es la regulación cultural y social, así como el control social, en gran medida inconsciente, los que conforman la conciencia de los sujetos. En esta situación el objetivo debe consistir en la eliminación de las patologías sociales que esos esquemas generan a través de la hermenéutica del mundo de la vida cotidiana y la crítica reconstructiva. Quienes defienden este modelo consideran que una tarea así no se puede alcanzar con el modelo cognitivo de corte cibernético. Lo rechazan por abstracto e irrealista ya que descuida los diferentes contextos empíricos, la historia cultural del sujeto y de los grupos sociales, y porque explica la conducta a través de mecanismos abstractos desligados de la vida, separados artificialmente de las vivencias y de la experiencia real. Pero paradójicamente se puede decir que la esencia del modelo es idéntica. En efecto, uno de los axiomas básicos del actual modelo vigente en la neurociencia se condensa en esta fórmula: nues- 
tro cerebro está construido socialmente. Cambian los términos, se usan categorías diferentes y los espacios de investigación son distintos, pero el modelo es el mismo. La persona es, según estos planteamientos, un ser socializado que se somete voluntariamente a la presión social porque no puede escapar de la fuerza socializante que ejerce sobre su yo la cultura que necesita compartir con los miembros del grupo. La conciencia de identidad es un atributo del individuo pero el proceso de producción de esta conciencia es social en la medida en que se basa en significaciones sociales de rasgos individuales y colectivos (Pérez Agote 2006: 420; Quante 2007: 57; Owens y otros 2010: 478).

Ambos esquemas de interpretación adolecen del mismo error: la eliminación de la conciencia individual, el olvido de la valiosa singularidad de los pensamientos y emociones del sujeto, es decir, de la persona singular y única que somos. Y la raíz de este fallo es la misma: el abandono del enfoque ontológico. En su lucha contra el fantasma de un sujeto sustancial o trascendental han prescindido de su consistencia entitativa; en su rechazo de una visión esencialista de la identidad han prescindido del ser que somos; en su crítica al moderno hiperindividualismo han negado la existencia del sujeto; y en la dura batalla contra el espantajo de una conciencia autónoma y, totalmente transparente a sí misma, desposeen a la persona de conciencia propia. Y es la pérdida de su consistencia ontológica la que termina por reducirlo a una máquina desprovista de estructuras cognitivas o programas propios con contenido informativo que sirven de soporte a la conciencia de las personas y las capacitan para discriminar y seleccionar los estímulos para dar respuestas adecuadas. Tan arraigado está el modelo que ya no produce extrañeza una reciente tesis en la que se afirma que el verdadero y único reto que tiene la antropología es desvelar la cultura emocional y sentimental del "autómata social" (Farrugia 2009: 284). Se podría recordar, ciertamente, que los antropólogos siempre han subrayado que la identidad personal no está determinada por las estructuras sociales y que es precisamente el vínculo social lo que nos singulariza en la medida que ofrece posibilidades, recursos, señas de identidad, etc., elementos todos ellos que hacen posible la construcción del yo personal. Pero ha sido la exagerada exaltación del yo institucionalizado la que ha conducido a la pérdida de la sensibilidad epistemológica por la verdadera identidad de las personas. Se puede apreciar en este reciente texto:

Por lo demás, cuando no se cree más en las instituciones, igualmente tampoco se cree en los individuos. El valor de un individuo, el hecho de que él viva o muera no tiene ninguna importancia. Porque un individuo no existe más que sostenido por las instituciones. Solo, él no es nada (Birman 2011: 110). 
La tesis del autómata social es la formulación cruda y desnuda del modelo que rige en el campo de las ciencias sociales. Como botón de muestra expondré la solución que se propone a las denominadas crisis de identidad - exilio, divorcio, despido, paro, enfermedad prolongada, jubilación, decepciones personales, precariedad económica, exclusión social, etc.- y que según muchos autores son las verdaderas causas del sufrimiento social que padecen los miembros de las sociedades desarrolladas. Son momentos en los que la persona necesita estar a solas consigo misma para reconquistar o reconstruir su identidad personal. Y la solución que se propone para ese problema es el repliegue sobre la identidad primitiva que hunde sus raíces en los lazos primarios, familiares y comunitarios. Y es que cualquier crisis identitaria remite a los vínculos primeros de la existencia que conforman el triángulo edípico, a las fuentes primarias del yo, al nosotros fusional y comunitario (Dubar 2000: 229; Gergen 2009: XVI; DeGloma 2010: 82). No sirve la cura psicoanalítica a través de la cual se pretende reconstruir el núcleo íntimo de la persona, porque el terapeuta no puede acceder al fondo insondable de la conciencia. La única alternativa que tiene la persona para superar estas disfunciones sociales es encontrar el camino seguro que le lleve al encuentro con el otro personalizado. En suma: la solución se halla en los otros porque son los otros los únicos que nos pueden ayudar a reconstruir la identidad en crisis. Y no debería sorprendernos esta propuesta: la persona solo existe en la medida en que asume y se inserta en las estructuras del grupo, ya sean de tipo comunitario o societario. Es ésta, por otra parte, la consecuencia lógica de un proceso de desintegración de la persona que, como resultado de su trabajo de campo en Brasil, ya anunciara y anticipara un clásico de nuestra disciplina para la teoría antropológica: la entropología. "Plutôt qu'anthropologie, il faudrait écrire "entropologie" le nom d'une discipline vouée a étudier dans ses manifestations les plus hautes ce processus de désintégration" (Lévi-Strauss 2008: 444). Merece la pena analizar el proceso de cómo se ha llegado a cumplir este pronóstico que, a simple vista, choca con toda la tradición antropológica en la que se ha sometido a una acerada crítica, tanto el estructuralismo, como su versión y transformación en post-estructuralismo.

\section{LA CATEGORÍA DE PERSONA EN ANTROPOLOGÍA}

En esta disciplina se resalta el papel de la acción individual y se valora la relevancia de los métodos cualitativos para comprenderla. En efecto, la etnografía se centra en las personas concretas, en los datos y detalles que nos cuentan de sus vidas. Se recuerda a los investigadores la necesidad de 
buscar formas y fórmulas —desde la teoría, la epistemología y la metodología antropológicas - que permitan la inclusión de los individuos y poder convertirlos en protagonistas y agentes de su historia. Pero, paradójicamente, estos ideales han desembocado en la disolución de la identidad personal. En la mayoría de estas investigaciones se concluye que las personas solo se realizan como tales cuando aceptan y se integran plenamente en una cultura que les dota de un sistema de creencias y valores, una lengua, unas costumbres, una historia, una visión de futuro, etc., pero que también se les impone. Estos presupuestos implican que el sujeto se identifica plenamente con sus roles sociales y como consecuencia conllevan su disolución en la red de relaciones sociales. En este enfoque se prima la importancia de la sociedad y la cultura en la conformación de la identidad individual. Lo que llamamos persona de hecho se disuelve en el nosotros y, por tanto, la identidad personal tiene una naturaleza social (Strathern 2011: 100-101; MacKinnon y Heise 2010: 15; Burton 2007: 45; Wagner 2001: 31).

La antropología nació bajo el manto de este axioma, se conformó y sigue estando configurada por el funcionalismo sociocultural en sus diferentes pero también sutiles variantes. En efecto, al iniciar el trabajo de campo ya se da por hecho que cada miembro del grupo social debe ser considerado, porque ellos también se consideran a sí mismos como seres en relación, que poseen unos modos de ser y pensar que están configurados por la interacción que mantienen con los otros, y estas relaciones no solamente contribuyen a configurar la identidad, sino que la crean. Es cierto que se proclama el valor de lo individual pero quien lo proporciona, lo garantiza, lo funda y lo crea es la comunidad de pertenencia. Esa es la posición hegemónica, tanto en el funcionalismo puro, como en su versión estructuralista, en la interpretativa y en la post-estructuralista. Es una de las tesis más repetidas en antropología: la identidad personal es el resultado de la sociedad y la cultura en la que uno le ha tocado vivir (Lévy-Bruhl 1927: 70; LéviStrauss 1977: 332; Luckman 1979: 175; Douglas 1983: 46; Heritier 1996: 281; Ingold 1998: 745). Tanto el trabajo de campo en sociedades tradicionales o modernas como los estudios comparados que se han realizado a partir de esas investigaciones han partido y además han desembocado en una tesis comúnmente aceptada: la identidad personal se crea y, en consecuencia, se diluye en la trama de relaciones y significados sociales.

Todas las culturas han elaborado una reflexión comparada, contrastada y a veces contradictoria, acerca de las nociones de individuo y persona, y todas se han encontrado con la misma dificultad: la noción de individuo puede disolverse por exceso o por defecto de sentido (Augé 1993: 231; Fortes 1987: 250). En el campo de la antropología, por influencia del positivismo y del marxismo, la persona queda disuelta en la trama de las relaciones 
sociales. Un hito histórico clave en este proceso de disolución de la identidad personal es la conferencia que dictó M. Mauss en 1933 en Londres. En ella aborda directamente el tema de la persona. Mauss rechaza el sesgo ontológico de este concepto como categoría de pensamiento e interpretación antropológica y sostiene que la investigación se debe concentrar en el estudio de cómo se forma y se ejerce la personalidad de los individuos en diferentes culturas. Sostiene que el trabajo de campo se debe focalizar en la comprensión del papel que el individuo desempeña dentro de la sociedad a la que pertenece y en la manera cómo la cultura del grupo conforma sus modos de ser y pensar, es decir, cómo le convierte en persona. La antropología debería ser una especie de historia social de las diferentes formas de llegar a ser persona. Afirma que lo que el antropólogo debe hacer es describir e interpretar los variados papeles que el sujeto como persona ha desempeñado a lo largo de la historia en diferentes tipos de sociedades (Mauss 1968: 335). La misión de la antropología consistiría en crear un atlas de las diferentes maneras de ser persona en culturas distintas a la propia. Y a este programa de investigación se adhirió otro autor clave en el proceso de disolución de la categoría de persona, C. Geertz, quien ajustó sus investigaciones a ese planteamiento. Debemos reconocer la importancia de sus densas descripciones sobre los modos de ser persona en Bali a través de sus diferentes expresiones sociales que nos permiten identificar a una persona como individuo único: nombres personales, nombres según el orden de nacimiento, términos de parentesco, tecnónimos, títulos de status y títulos públicos (Geertz 1973: 368). Pero mucho más importante que su trabajo de campo son dos supuestos teóricos que han pasado a formar parte del acervo de la teoría antropológica. De una parte, rechaza el concepto clásico de naturaleza humana para concebir al hombre como un ser puramente cultural. Para huir del naturalismo de la teoría evolucionista sostiene que la cultura no se sobreimpone a la dimensión biológica, sino que se superpone. Tanto filogenética como ontogenéticamente los humanos son construcciones culturales que en manera alguna poseen un sistema endógeno que les permita actuar autónomamente. Y de otra parte, rechaza el modelo cognitivo, para decantarse por un modelo conductista del sujeto. Solamente se puede estudiar lo que se puede observar. El ser humano aprende de su medio social por medio de mecanismos conductistas, del tipo estímulo-respuesta y unos pocos principios generales más. Se concibe al ser humano como un animal inserto en tramas de significación que él mismo va creando. Este conjunto de significados constituye el marco y el horizonte en el cual existimos y nos desarrollamos y, en consecuencia, el verdadero espacio en el que se puede comprender el modo en que se puede llegar a ser persona. Su mente no está en su propio cuerpo, sino en el mundo social en el que vive. En suma: 
nuestras mentes son receptores pasivos de la cultura que compartimos con los miembros de nuestra sociedad (Geertz 2000: 205). Persiste hasta nuestros días este modelo, actualizado recientemente y condensado en esta tesis: la intencionalidad colectiva, tanto desde un punto de vista filogenético como ontogenético, constituye el origen de la humanidad y es además la que crea la intencionalidad individual. Lo que hace singular al ser humano, la clave de nuestra específica naturaleza se halla en la intencionalidad compartida que hace posible la aparición de formas únicas de cooperación que no poseen los animales (Tomasello 2010: 17-18).

Esta apretada síntesis nos permite apreciar que ha sido la pérdida de la consistencia ontológica de la persona, iniciada por M. Mauss, la que ha tenido como consecuencia la disolución de la identidad personal. Cuando se prescinde de las constantes antropológicas y se prima el poder ilimitado de la cultura y de las culturas, el enfoque ontológico nos indica la necesidad de poner los pies sobre la tierra. Nos obliga a partir de los hombres concretos cada uno de los cuales tiene una consistencia entitativa que podemos percibir en forma de propiedades físicas y estructurales que condicionan su vida dentro del medio social, físico y cultural. Nos indica la necesidad de tener en cuenta el barro de que estamos hechos, el tipo de criatura que somos, que remite a las constricciones naturales que impone el diseño estructural y funcional del cuerpo que somos. La categoría de persona obliga a un enfoque realista del sujeto porque permite comprender el concreto antropológico de manera más fértil. Para poder escapar de las constricciones que nos impone la estructura psicobiofísica que somos así como del control y la presión que nos impone el contexto sociocultural para lo que podemos llegar a ser, se necesita una teoría del sujeto que los conjugue y al mismo tiempo los supere (Bunia 2010, 571). Para poder construirla se necesita reflexionar previamente sobre estas cuestiones: ¿qué modo o tipo de ser es ese sujeto singular que puede distanciarse y al mismo tiempo disponer de su propio cuerpo? Es decir, tenemos que tener en cuenta lo que somos, dar cuenta de la estructura entitativa que configura nuestro ser. Y serán las ideas de uno de los padres del concepto de identidad las que nos pueden servir de hilo conductor para construir un modelo no excéntrico: Erving Goffman.

\section{ONTOLOGÍA DE LA PERSONA}

Este pensador es conocido por sus estudios sobre la interacción simbólica, la influencia de los significados y los símbolos sobre la acción y la interacción humana, los encuentros cara a cara en grupos reducidos, etc. El 
suyo es un enfoque dramático o un análisis dramatúrgico de la vida cotidiana en cuyos escenarios Goffman acierta a describir cómo las personas se presentan ante los demás como personas, es decir, cómo expresan su identidad personal. Se trata de una microsociología sustentada en un modelo teórico que consta de tres variables: persona, interacción social e institución (Goffman 1959: 242). La descuidada y olvidada dimensión de identidad personal — se resalta la identidad del yo y la identidad social- es la base de la interacción social y este sociólogo la fundamenta en un concepto ontológico de persona. Lo podemos ver con claridad en este texto:

Aquello que diferencia a un individuo de todos los demás es la esencia de su ser, un rasgo general y nuclear de su persona que lo hace completamente distinto $-\mathrm{y}$ no solo en cuanto a su identificación— de quienes más se le asemejan (Goffman 1972: 77).

La referencia a este gran investigador es intencionada. Son muchos los científicos sociales que consideran que el marco teórico propuesto por él sigue siendo válido para explicar el tema de la identidad digital. Y si repasamos la literatura reciente constatamos que en él se apoyan muchos investigadores para tratar de explicar este nuevo tipo de identidad virtual que se construye en el ciberespacio (Escobar y Román 2010: 1; Georges 2009: 2; Roesler 2008: 427). Merece la pena entender por qué se han recuperado sus ideas para explicar la identidad digital. Ello nos ayudará a entender por qué es necesario mantener el enfoque ontológico.

El clima intelectual en el que nos movemos actualmente está configurado por la cosmovisión evolucionista. Y debemos reconocer que la aparición de la teoría evolucionista y la definición biológica del ser humano como Homo sapiens supuso un avance decisivo en la comprensión de la unicidad del hombre. En este contexto se puede situar una reciente definición de la "“identidad personal" como el conjunto de condiciones que permiten a un individuo considerarse un ser humano igual y diferente de los demás: individuo de una especie distinta de cualquier otra, es decir, persona humana o moral" (Núñez Ladezéve 2005: 77). Este enfoque es sumamente valioso pero necesita ser proseguido. Y debe serlo porque se elude y no se aborda directamente la cuestión de en qué consiste ser persona, es decir, el problema de su realidad. Sin embargo, la aparición de la denominada identidad digital propiciada por Internet nos está obligando a retomar la cuestión ontológica.

De un instrumento utilizado en el solitario anonimato ha pasado a convertirse en un medio que incide en cuestiones relacionadas con la naturaleza y la identidad humana: quiénes somos, qué sentimos en relación a nosotros mismos y cómo nos comportamos unos con otros. Mientras las viejas teorías de la socialización en lí- 
nea se derrumban, brotan nuevas preguntas en torno a la relevancia psicosocial del uso de redes sociales (Disalvo 2011: 61).

El impacto del ciberespacio en nuestras vidas, la potencia ontológica de las nuevas dimensiones virtuales que configuran el ciberespacio, la omnipresente dicotomía de lo real/virtual ha obligado a volver a pensar el estatuto ontológico de la realidad, a reflexionar sobre los rasgos esenciales que caracterizan lo que consideramos real. Las nuevas tecnologías de la información y comunicación, cuya plataforma es Internet, han liberado a la interacción de los requisitos de co-presencia física. Estas tecnologías han ampliado y consolidado el conjunto de redes sociales que están haciendo posible la construcción del yo digital en el que se disuelve y confunde lo personal y lo virtual. Pero inmediatamente surge la pregunta: ¿qué tipo de realidad posee? ¿Cómo de real hay que considerar a este yo virtual? ¿Es esta forma de identidad una característica intrínseca de la persona, o una pura ficción creada ilusoriamente por el conjunto de individuos que conforman la red social en la que se participa? Se necesita entender el tipo de realidad en la que consiste la identidad digital/virtual. Ya no nos podemos contentar con la idea de que lo real y lo irreal son cuestiones básicas en la construcción o convención social (Tubau 2011: 307; Sloterdijk 2010: 166).

Precisamente de lo que se trata es de entender el tipo de realidad de la identidad digital y la naturaleza de la convención social que hace posible su surgimiento. El sujeto se percibe como cuerpo porque no existe otra forma de existencia, pero al mismo tiempo se siente y vive en realidad como una idea, una mente, un pensamiento colectivo. Para explicar esta paradoja ha habido que volver a la ontología, es decir, se ha producido un giro ontológico. Pero este retorno a la ontología renuncia de manera explícita a las aportaciones del pensamiento clásico. Sin embargo, conviene hacer ver que esos requiebros sucumben ante el cientificismo larvado que configura la cultura occidental: pensar que la física-matemática actual ya ha conseguido una explicación satisfactoria y definitiva de la realidad. Sus partidarios se alinean con los que están totalmente convencidos de que la explicación y comprensión definitiva de cualquier tipo de realidad se alcanzarán con el desarrollo del enorme potencial teórico que proporciona la mecánica cuántica. En esta teoría se halla para ellos el fundamento explicativo de la base material a la que hacen referencia. Y debemos reconocer que es la mejor descripción de la realidad que poseemos, que ningún experimento realizado hasta ahora ha podido destronarla y que, por tanto, estamos obligados a reconocer sus aportaciones. Pero, hoy por hoy, esta ontología fundamental es incapaz de aportar algún tipo de luz sobre, por ejemplo, un rasgo esencial de la persona: el amor (Chudnovsky y Tejada 2011: 206; 
Schroll 2010: 6; Baruss 2010: 213). Solamente proporciona definiciones operativas y funcionales y, como señalara Einstein, es una teoría incompleta. En este estado del saber únicamente podemos avanzar manteniendo los aspectos positivos del modelo clásico. Es la única vía que nos ayuda a evitar la caída en una ontología procedimental que solamente explica la realidad de manera funcional; una ontología nihilista y abstracta en la que se difumina la identidad personal.

El mundo de la vida cotidiana constituye el escenario en el que la persona produce y al mismo tiempo es producida por la cultura de su propia comunidad. Se puede aceptar que la identidad personal es el fruto de una constante negociación entre las reglas sociales interiorizadas y las respuestas del núcleo subjetivo más profundo, pero en manera alguna prescindir de ese núcleo estable en la medida en que de él dimana la identidad de las personas. Estructura y proceso están acoplados y se complementan. Por ello es falso explicar la identidad del ser humano única y exclusivamente a través del proceso sociocultural en el que se conforma, es decir, no podemos soslayar la cuestión de qué es la persona en sí misma. Constituye una ganancia absoluta del saber el que todas las disciplinas, de manera implícita o explícita, se apoyen en una concepción sobre qué tipo de realidad es la cosa que abordan. La razón de esta necesidad es simple: la identidad tiene que ver con lo que soy y no con lo que desearía ser, aunque lo que desee ser influya en mi identidad, en lo que soy, o termine formando parte de mi identidad. El desafío que presenta la vida consiste en aprovechar las posibilidades que brindan los genes y el entorno físico y social. La habituación correcta es la forma adecuada que tenemos para conformar nuestras disposiciones. Es el mecanismo mental que hace posible la revisión, respaldo o el rechazo de nuestros modos de ser, pensar y actuar. En nuestro mundo líquido e hiperindividualista se piensa que la práctica del arte de la vida consiste en un estado de transformación permanente, en redefinirse perpetuamente, en dejar de ser lo que hemos sido hasta ahora para convertirnos en otra persona. Es un vano intento, pues podemos cambiar la forma de nuestro molde pero jamás desprendernos de él, dejar de ser lo que somos para llegar a ser por arte de magia la persona que imaginamos que podemos llegar a ser (Bermúdez de Castro 2010: 227; Díaz Viana 2009: 31).

Se pueden dar todo tipo de interpretaciones sobre el dato evidente de la denominada identidad numérica, recalcar la insuficiencia de la visión esencialista y estática del sujeto; uno se puede escudar en el hecho de que el concepto de sujeto como categoría antropológica es de creación reciente, e incluso hablar despectivamente de la escalada ontológica que supondría volver a una metafísica de corte aristotélico-tomista. Es cierto: la ciencia actual no puede avalar la idea de un desnudo substrato que sirva de 
soporte a las propiedades de la persona. Ello, sin embargo, no implica que debamos renunciar a las aportaciones del pensamiento clásico. Ontología y ontogénesis no son perspectivas incompatibles sino complementarias (Bickhard 2009: 3). En efecto, es posible recuperar el enfoque ontológico y al mismo tiempo avanzar en el conocimiento del status ontológico de la persona - evitando el Scila del esencialismo y el Caribdis del posconstructivismo- apoyándonos en la fértil idea de invarianza estructural. Esta noción facilita una descripción científica de los sistemas biológicos, ya que es capaz de distinguir y conectar el aspecto invariante de las dinámicas vivientes y el aspecto variable, la estabilidad y la flexibilidad estructural. Es ésta una propiedad de la morfología intrínseca del sistema humano que fundamenta una concepción consistente y robusta de la identidad personal. La estructura puede variar de organismo a organismo, pero la organización es esencialmente la misma y constituye el mecanismo que garantiza la identidad del sistema visto como un todo. La organización es el invariante de la dinámica de los sistemas biológicos, el complejo unitario de relaciones que constituye la identidad de cualquier ser vivo (Damasio 1999: 142).

Es cierto que todos nacemos con una identidad dada, aunque muy precaria pues necesitamos del vínculo maternal y familiar para sobrevivir y desarrollarnos como personas. Pero en germen y en potencia se halla esa unicidad totalizadora que marca, aunque en manera alguna determina, la línea vital que debe construir creativamente cada persona a lo largo de su vida. En efecto, es cada persona la que por su propia iniciativa y siguiendo su propia dinámica la que se despega de este suelo acogedor (Morin 2011: 287). Y esta dinámica se tiene que ejercer en muchos momentos de la vida, pero especialmente cuando se presenta un problema que por sí mismo hay que resolver: ¿quién soy yo? Es el clásico y eterno problema de la identidad: cómo se ve la persona a sí misma y como piensa que los demás la perciben. No es una cuestión que nos tengamos que plantear cada mañana, pero en muchos momentos tenemos que afrontar el problema de la identidad personal "La persona necesita saber acerca de sí misma en cuanto persona, ya que es constitutivamente problematicidad y reflexividad. La vida misma conduce al planteamiento por el ser-persona" (Aguirre Oraa 2011: 109).

En efecto, estamos obligados a hacernos en muchas ocasiones esta pregunta: ¿somos lo que somos en un sentido absoluto o son los demás los que nos tienen que decir, de vez en cuando, quiénes somos? Y son las personas las que se atreven a superar el miedo a ser diferentes, a rechazar el dualismo identitario por el que uno se reconoce a sí mismo en el otro como enemigo. Es difícil atreverse a distinguirse. El trabajo de campo demuestra que la capacidad de la gente para pensar y actuar por sí misma varía en función de su talento y formación. Pero también constatamos que personas 
adultas y normales cuestionan, desafían y se oponen a lo que se les ha enseñado o se les quiere imponer. A pesar de que en algunas ocasiones las circunstancias particulares tal vez no alientan a una persona a hacer ese cuestionamiento, la capacidad de dudar y de cuestionar se ejerce. En muchos contextos nos tenemos que enfrentar al duro dilema de si se puede ser sin pertenecer y en consecuencia afrontar el reto de salir al mundo a buscar y construir nuestra propia identidad, o si, por el contario, debemos aceptar, adquirir y actuar con una identidad que esta fijada o es definida por los códigos de conducta que se nos quiere imponer. Este tipo de dilemas que surgen en diferentes ámbitos de la vida individual y colectiva profesional, familiar, política, económica, etc. - ha obligado a recuperar el concepto de identidad personal (Aguiar y otros 2010: 273). Esa recuperación exige mantener las aportaciones de la visión clásica de persona que impide diluir al sujeto en su ambiente sociocultural. Engarza directamente con el enfoque ecosistémico, uno de cuyos principios básicos es que no se puede definir un sistema únicamente por lo que es, sino también por el entorno, ya sea físico, social o cultural. Este enfoque posibilita un conocimiento más fértil de la identidad y, sobre todo, una base sólida para la renovación y actualización de la categoría clásica de persona.

\section{PERSONA Y CONCIENCIA}

En el lenguaje corriente el uso del término persona no causa perplejidad pues se aplica a todos los seres humanos e incluso se ha consolidado la distinción entre persona física y jurídica. Es además una palabra de uso común en muchas disciplinas que indistintamente se sirven de los términos persona y personalidad. La dificultad aparece cuando se usa como categoría técnica y se trata de aquilatar el contenido y el alcance de esta categoría. Pero si abordamos esta cuestión lo primero que debemos señalar es que en el saber de nuestros días ya no produce extrañeza que volvamos a preguntarnos: ¿Qué es una persona? (Bayés 2009: 71; Lenclud 2009: 3; Raulin y Dussy 2008: 5). Pues bien, la respuesta a esta simple pregunta tiene dos caras, como nos enseñó E. Goffman, cuando una persona se presenta ante los otros les informa de lo que es — is - y de lo que ellos deberían —ought - ver en ese es (Goffman 1959: 13). Efectivamente, en esta cuestión el anverso remite a la cuestión ontológica: en qué consiste ser una persona; es decir, cuáles son los rasgos que nos permiten tener seguridad de que un determinado tipo de realidad que vemos o que nos apela puede ser llamada persona; el reverso remite a la cuestión sobre qué significa ser persona, es decir, qué valor debemos atribuir y cómo nos debemos comportar con un ser que se presenta ante nosotros como persona. 
A pesar de las reticencias e incluso del rechazo que provoca en los antropólogos abordar aspectos de orden ontológico se debe reconocer que topamos con una cuestión insoslayable. Se podría pensar que se trata de asuntos especulativos reservados a las discusiones que entablan los filósofos, identificarse con el camino iniciado por M. Mauss que prescindió del legado clásico para asignar su estudio al entonces incipiente pero pujante campo de la psicología, e incluso relegarlos al campo de la meta-antropología (Bidney 1970: 156; Blainey 2010: 116). Pero un repaso a la historia de la antropología nos muestra que esta cuestión acaba emergiendo y se tiene que afrontar. Tarde o temprano el antropólogo termina por comprometerse con una determinada concepción de la persona humana o tiene que decantarse por el factor que le confiere identidad y le diferencia de otros seres (Díaz Viana 2009: 28; Díaz de Rada 2010: 279-80; Ingold 2010: 6). Y lo hace porque se trata de una cuestión que afecta al núcleo de la teoría antropológica y al trabajo de campo que realiza cualquier antropólogo. En efecto, aceptemos la tesis oficial de que la identidad personal la confiere el grupo social, que la sociedad construye al individuo, es decir, que la conciencia individual es creada por la conciencia colectiva. Pues bien, los antropólogos que se escudan en esta tesis no solo defienden y practican el relativismo metodológico sino también el cognitivo: tantos modos diversos de ser persona como tipos diferentes de sociedades. Con palabras de un prestigioso antropólogo: "A diferentes culturas, diferentes racionalidades" (Lukes 2000: 16). Pero inmediatamente aparece esta pregunta: ¿Cómo es posible comprender y traducir tantos modos diferentes de ser y pensar? ¿Es viable que se puedan entender y dialogar personas que por principio operan con esquemas mentales tan dispares? Al responder a esta pregunta de manera insoslayable aparece el problema de lo universal y lo particular, y la tendencia actual es a complementarlos. Pero prescindamos de esta cuestión tan debatida y supongamos que sea plausible, como lo demuestra la gran cantidad de monografías que existen sobre los modos de ser persona en culturas tanto ajenas como propias. Entonces surge la pregunta: ¿cómo ha descubierto el antropólogo el modo en que las prácticas sociales - nombres, reglas de parentesco, roles, normas, tabúes, creencias, recetas, rituales, etc.- configuran la identidad de las personas? No cabe la menor duda de que se sirve de la conciencia social o memoria autobiográfica de las personas, pero ningún antropólogo ha intentado dar una explicación satisfactoria de cómo se realiza este proceso. Nadie ha explicado la tensión constitutiva entre la naturaleza natural y la naturaleza social, ambas integradas dialécticamente en la naturaleza del individuo. Se trata de un estudio necesario, porque es el único espacio desde el que se puede abordar el conocimiento de la racionalidad de los actos individuales que contribuyen a re- 
forzar el vínculo social y de aquellos que contribuyen a disolverlo (Bilbao 2007: XXI). Lo dan por hecho, pero nadie ha conseguido mostrar cómo se generan y cuáles son los esquemas mentales que conforman la mente de los agentes sociales que dan vida a la sociedad que el antropólogo toma como unidad de análisis. Se parte de teorías generales, se rechaza el determinismo sociocultural y se acepta que el proceso de construcción de la persona es un proceso constante de enculturación que se inicia con la socialización primaria y transcurre durante todo el ciclo vital. Para ir más allá de generalidades es necesario entrar en los recovecos de la mente humana para entender y explicar cómo se conforma este proceso. Podemos empezar con estas preguntas: ¿hay algún lugar en este proceso para los valores e intereses de los sujetos que son fabricados por la sociedad? ¿Tiene algún papel el sujeto individual en los cambios que se producen en todo tipo de sociedades? La pregunta es pertinente porque no existe sociedad alguna sin cambios y tampoco alguna en la que se niegue la identidad numérica, es decir, la distinción clara del yo y del tú (Lenclud 2008: 63; Ingold 1998: 844). Todas estas cuestiones se pueden soslayar, pero qué duda cabe de que el simple hecho de plantearlas y tratar de resolverlas permite progresar en el saber antropológico (Bloch 2009: 63; Bender y Beller 2011: 67). En todo caso, nos ayudan a evaluar lo que se sabe y no se sabe de las personas, a darnos cuenta de que es posible y además necesario seguir investigando para avanzar más allá del estado actual en el que se encuentra el saber antropológico. Este es un proceder necesario porque en las ciencias sociales se está extendiendo y calando la idea de que está agotada la fuerza y el predominio acrítico que siempre ha tenido el modelo culturalista (Lizón 2010: 412; Aguiar, Francisco y Noguera 2009: 443).

Si en el presente queremos salir de esta situación para avanzar en el esclarecimiento de la naturaleza del sujeto humano necesariamente nos debemos situar en las actuales coordenadas del saber, como siempre se ha hecho en antropología. Para iniciar esta tarea, pienso que el punto de apoyo deben ser las ideas propuestas por G. H. Mead. En su modelo podemos encontrar el enfoque ontológico que E. Goffman insinuó pero no desarrolló. Entiende aquel pensador que la pregunta clave es ésta: ¿qué tipo de organismo es el ser humano para que en él pueda emerger la conciencia? Sitúa esta pregunta en el campo de la ontología. Aclara que ni la física de Aristóteles ni la mecánica de Newton sirven para conseguir ese objetivo. Es la nueva física basada en la teoría especial y general de Einstein y la mecánica cuántica de donde debemos partir. Pero reconoce que esta ontología fundamental no sirve para aclarar y responder a la pregunta:

Ese reflejo de realidad en las identidades que busca el método científico sólo conduce a un sólido parmenídeo. Este hecho se vuelve particularmente evidente 
cuando consideramos lo que llamamos significado de las cosas. Están éstos inextricablemente entretejidos con lo que debe designarse con el término conciencia; y sin embargo esos significados son la verdadera naturaleza de los objetos científicos. La otra razón es que eso que llamamos conciencia está siendo incorporado en la esfera de la ciencia biológica. Ya no cabe seguir dejando la mente fuera de la naturaleza (Mead 2008: 329-30).

Pero esta reflexión no es estéril porque le sirve para fijar el espacio o nivel de realidad donde apoyarse para avanzar en el esclarecimiento de la naturaleza de la conciencia. Sus categorías de organismo, entorno y emergencia nos conectan directamente con los actuales avances del saber. En efecto, para proseguir su tarea el marco teórico más fértil que poseemos en la actualidad es el que nos ofrecen las ciencias de la complejidad. Aboga por concepciones totalmente nuevas, otros modos de pensar, modelos explicativos e interpretativos que puedan abrir caminos hacia la explicación de la realidad compleja que cada uno de nosotros somos (León y Sanjuán 2009: 60; Reza 2010: 125). Contiene un programa de investigación en el que se mantiene la tensión ontológica, ya que está orientado y enfocado a fijar los elementos de los que consta un sistema, las relaciones que comporta, los mecanismos que generan los cambios y las adaptaciones que realiza, las fronteras pero también las conexiones que mantiene con el medio, tanto físico como sociocultural. Desde un punto de vista epistemológico promueve la construcción de modelos en los que se pretende garantizar la validez de las variables que se usan, la interpenetración conceptual que viene exigida por la variedad de estudios especializados, los niveles desde los que se puede estudiar, etc. Desde un punto de vista evolutivo propone una visión emergentista de la persona que no claudica ni sucumbe ante la fascinación que consigue en el saber actual el reduccionismo de las ciencias empíricas - somos nuestro cerebro- ni ante el determinismo de las ciencias sociales - la cultura crea nuestra propia conciencia. No se puede considerar como una teoría definitiva, pues se reconoce la necesidad de superar la ambigüedad que contienen muchas de sus categorías: aquilatar el concepto de complejidad, fijar los niveles de organización, precisar la idea de auto-organización, encontrar las leyes de la emergencia, aplicar la idea de causalidad circular, desterrar definitivamente el reduccionismo, etc. (Ellis 2009: 33). Pero a pesar de estas limitaciones es un enfoque fructífero, ya que nos permite progresar en el conocimiento de la naturaleza de la persona y, sobre todo, es valioso porque recupera y fundamenta la identidad personal en una ontología consistente. En él nos podemos apoyar para renovar y formular una visión reticular del concepto clásico de persona para, de esta manera, recuperar la importancia de la identidad personal.

En este nuevo contexto debemos valorar y aprovechar las aportaciones de antropólogos que se han esforzado por establecer puentes con el saber 
de nuestros días para construir una antropología integral. Siguen esta vía porque están convencidos de la validez de una idea que repetía constantemente Caro Baroja: en el orto de las Ciencias los saberes son coherentes y se relacionan entre sí, no porque haya quienes los estudien de modo interdisciplinario, sino porque lo son en su realidad total.

Y esto es así porque la ciencia significa búsqueda, lo que supone hallazgos, pero también aparición de nuevos interrogantes. En cuanto al acercamiento entre científicos y místicos, etcétera, podríamos decir que esto sucede porque ante nuevos descubrimientos y nuevos horizontes la complejidad es mayor, y por tanto, se hace prácticamente indispensable la colaboración entre unos y otros (Caro Baroja 1995: 7).

En estas ideas (Caro Baroja 1991: 226) nos podemos apoyar para conectar e integrar las aportaciones de diferentes disciplinas, tanto naturales como humanas y sociales. Afrontan el reto interdisciplinar que les lanzan tanto la nueva psicología evolucionista como las ciencias neurocognitivas, y no se esconden ante este desafío porque son conscientes de que los descubrimientos de las dos últimas décadas han alterado el paisaje intelectual en el que actualmente nos movemos. Además son muchos los que se sirven de las aportaciones que ofrece la nueva teoría de los sistemas complejos. Un concepto clave de este enfoque es el de sistema complejo adaptativo. Es una categoría que está sirviendo para progresar en el saber y por ello no debería extrañarnos que sean numerosos los antropólogos que se hayan adherido a esta nueva orientación (Ellen 2010: 395).

En este modelo la persona ya no se puede concebir como una sustancia racional. Pero la fundamentación antropológica de este concepto sí conecta con la tradición clásica. No se apoya en su visión sustancial, pero tampoco desprecia o prescinde de la impronta del patrimonio cultural acumulado, ni de la fuerza homogeneizadora que tiene en el presente la cultura de la sociedad neoliberal, y tampoco de la capacidad de emancipación de las utopías sociales. Se apoya, sin embargo, en el pensamiento clásico, puesto que su punto de referencia es el ser total del hombre. Pero es también nueva y renovada, puesto que, para avanzar en la comprensión de esa totalidad, se sirve del concepto de sistema complejo adaptativo. Ofrece una visión del sujeto que remite a una ontología y no lo reduce a simples determinaciones socioculturales. Su aceptación obliga a recuperar la importancia de su estructura entitativa, a retomar la cuestión de la unidad y la unicidad del sujeto, a reconocer la importancia de la identidad personal para poner límites a la excesiva fragmentación y sometimiento de la persona por parte de la estructura social. Su punto de partida es el reconocimiento de que, como todos los seres vivientes, somos seres físicos constituidos por partículas elementales. No solamente desarrollamos nuestra vida en un universo físico sino 
que también procedemos de él y su organización físico-química está constitutivamente en nosotros. Es la continuidad física de las personas la que somete a las partes a la hegemonía de un sistema. En efecto, nuestra identidad es lo que somos y lo que pensamos sobre quienes somos; somos una entidad completa e independiente. Y es la conciencia, el sentimiento y la determinación que cada sujeto tiene de esa mismidad corporal lo que le convierte en un tipo especial de sistema y la que da consistencia, coherencia y continuidad a su trayectoria vital (Elias 1991: 251; Romagnoli 2010: 309).

Esta visión renovada nos permite describirla como un sistema abierto formado por elementos y relaciones que tiene un dentro y un fuera, un núcleo que lo conforma, y está influido y cambia con los procesos internos y por sucesos externos que le afectan. Pero estas constricciones a las que está esta visión sometida constantemente pueden en cierto grado ser superadas por la capacidad que le proporciona su conciencia reflexiva. En este marco ontológico se sustenta la sugerente y fértil idea de una coemergencia del sí-mismo autónomo y el medio que sirve de base para fundamentar y además avanzar en la comprensión de la persona humana así como de su autonomía y compleja identidad (Juarrero 2009: 97; Duval y otros 2009: 17). El sistema y su relación con el entorno crean una entidad en continua evolución, única e individual, pero una individualidad que se transforma constantemente. Esta totalidad es la base de nuestra compleja identidad personal.

La persona se concibe como la unidad de conciencia que persiste a lo largo del tiempo. Es algo que se siente como un sí-mismo estable en la medida en que se recuerda que lo experimentado ayer, lo experimento hoy y puedo anticipar que también lo experimentaré mañana. Para unificar esta serie temporal de experiencias vitales que conforman la vida cotidiana de cualquier sujeto se necesita la conciencia del yo que sirve de base a la identidad persistente de uno mismo. Esta entidad remite a un sistema dinámico y complejo sobre el que se conforma la identidad personal y cuyo núcleo es la conciencia. La conciencia es aquella facultad que permite focalizar nuestra atención sobre nosotros mismos y nuestros propios pensamientos y sentimientos (Álvarez Munárriz 2005: 13). Tiene una base cerebral, pero su estructura y funcionamiento está configurada por la experiencia del medio global en el que se ejerce. Conforma un espacio caracterizado por formaciones de bucles donde, por un lado, la conciencia corporeizada de la persona construye el mundo en su interacción con él y, por otro, esa misma mente consciente es construida en la interacción con el medio ambiente natural, personal, social y cultural. Constituye el núcleo del ser psicobiofísico que somos y en última instancia, genera una identidad personal, ciertamente compleja y relacional, pero robusta, consistente y creativa: 
¿Y cuál es el último talento que la conciencia ha concedido a los seres humanos? Tal vez la capacidad de navegar en un futuro por los mares de nuestra imaginación, guiando la embarcación de la identidad reflexiva hasta un abrigo seguro y productivo. Este talento, el mayor de todos, depende una vez más de la intersección del ser sí mismo y la memoria. La memoria, templada por la sensibilidad personal, es lo que permite a los seres humanos imaginar el bienestar, tanto individual como social, e inventar las maneras y los medios para alcanzar y magnificar ese bienestar (Damasio 2010: 443).

\section{A MODO DE CONCLUSIÓN}

En las sociedades desarrolladas de Occidente es el valor y la dignidad de la persona la que funda la sociedad. La etnografía demuestra que existen otras sociedades en las que ni se acepta ni se respeta este principio, pero también constata que tanto unas como otras están formadas por personas. Son personas las que conforman las familias, son personas las que crean y dan vida a las instituciones, son personas las que manejan los hilos del poder del Estado, son personas las que poseen y controlan ese enorme entramado de poder económico y militar, y son personas las que están dirigiendo la geopolítica y el destino de este planeta cada vez más globalizado. $\mathrm{Y}$ es únicamente la toma de conciencia por parte de las personas de esta situación de donde puede surgir la fuerza y el impulso para cambiar el estado actual de cosas que, tanto tirios como troyanos, consideran inviable e insostenible. Esta toma de conciencia hace que el sujeto deje de ser mero objeto de procesos, sistemas o estructuras para devenir persona. La apuesta por la persona deja en un segundo plano el tópico de qué puedes hacer tú por este o aquel ideal social para no ser engullido en la inteligencia colectiva. Incita, por el contrario, a que cada persona se plantee estas preguntas: ¿qué haces, cómo lo haces y para qué lo haces? Es simplemente una invitación a seguir el consejo del oráculo de Delfos: "conócete a ti mismo". Esa arriesgada tarea no se consuma ni consume el interior de uno mismo. La realización consciente de ese proyecto vital desprende una fina lluvia que nos empapa, pero que también riega esas parcelas de la sociedad en las que brota, crece y madura nuestra compleja identidad personal.

Esta apuesta por la persona subraya la importancia que el individuo tiene en la formalización y creación de una nueva cultura: la persona que piensa y decide por sí misma. Entiende que es posible el inicio de una nueva civilización en la que las personas deben ser los actores fundamentales en sus diversas encarnaciones: ciudadanos, inmigrantes, obreros, encargados, ejecutivos, patrones, etc. A pesar de las desestabilizaciones y presiones a los que están sometidos en la democracia neoliberal, el compromiso de la persona es la única vía a seguir en este mundo cada vez más globalizado. 
En esta arriesgada apuesta no debemos ignorar la dificultad que entraña el que los sujetos carentes de poder puedan hacer historia en un mundo donde los actores poderosos tienen permitido legalmente acaparar cada vez más derechos, más riqueza y más poder, mientras las masas de los sujetos en desventaja crecen de manera exponencial, al igual que sus desventajas; en un mundo en que se alza una nueva clase media privilegiada mientras la vieja clase media se empobrece, todo ello bajo un manto de legalidad. Pero en este mundo, multicultural y policéntrico que nos ha tocado vivir podemos iniciar el cambio si somos capaces de crear microrredes locales compuestas por personas auténticas: sujetos singulares con capacidad de actuar, de controlar su existencia, de ejercer su libertad, de elegir y construirse, de cumplirse. Y es que el cambio únicamente puede ser de tipo cultural: cambio en los modos de ser y pensar de las personas de carne y hueso. Tiene que ser la obra de nuevos actores, de personas capaces de inventar un sistema tecno-económico e institucional fundado sobre el derecho universal al conocimiento, el respeto y la creatividad. "Todavía se puede pensar que entre las fuerzas más vivas de la globalización y entre los sujetos personales singulares podrán ver luz nuevas y renovadas formas de vida democrática, algo realmente urgente" (Wieviorka 2011, 280).

\section{BIBLIOGRAFÍA CITADA}

Aguiar, F., A. de Francisco y J. A. Noguera 2009. "Por un giro analítico en sociología" Revista Internacional de Sociología 67 (2).

Aguiar, F. y otros autores 2010. "Personal identity: A theoretical and experimental analysis" Journal of Economic Methodology 17 (3).

Aguirre Oraa, J M. 2011. "Identidad humana y antropología. Somos los mismos, pero no somos lo mismo", en D. Bermejo (ed.), La identidad en sociedades plurales. Barcelona: Anthropos.

Álvarez Munárriz, L. 2005. "La conciencia humana", en L. Álvarez Munárriz (ed.), La conciencia humana: perspectiva cultural. Barcelona: Anthropos.

Álvarez Munárriz, L. y otros autores 2011. Conciencia e Identidad regional en la Comunidad de Murcia.

Murcia: Godoy

Augé, M. 1993. Genio del paganismo. Barcelona: Muchnik.

Baruss, E. 2010. "Beyond scientific materialism: toward a transcendent theory of consciousness" Journal of Consciousness Studies 17 (7-8).

Bayés, R. 2009. "Sobre la felicidad y el sufrimiento" Cuadernos de Medicina Psicosomática 89-90.

Bender, A. y S. Beller, 2011. "The cultural constitution of cognition: taking the anthropological perspective". Front. Psychology 2.

Bermúdez de Castro, J. M. 2010. La evolución del talento. Cómo nuestros orígenes determinan nuestro presente. Barcelona: Debate. 
Bertaux, D. 2005. Los relatos de vida. Perspectiva etnosociológica. Barcelona: Bellaterra.

Bickhard, M. H. 2009: "A process ontology for persons and their development" New Ideas in Psychology 27.

Bidney, D. 1970. Theoretical Anthropology. Nueva York: Schocken.

Bilbao, A. 2007. Individuo y orden social. Madrid: Sequitur.

Birman, J. 2011. "Entretien", en E. Enriquez (ed.), Désir et résistance, la construction du suject: contribution à une nouvelle anthropologie. Lión: Parangon.

Blainey, M. 2010: "The future of a discipline: Considering the ontological/methodological future of the anthropology of consciousness, Part II". Anthropology of Consciousness 21 (2).

Bloch M. 2009. "La mémoire autobiographique et le Soi. Pour une alliance entre sciences sociales et sciences cognitives". Terrain 52.

Bunia, R. 2010. "Das eingebildete Individuum". Merkur 64 (8).

Burton, J. 2007. "The anthropology of personal identity: intellectual property rights issues in Papua New Guinea, West Papua and Australia" Australian Journal of Anthropology 18 (1).

Caro Baroja, J. 1991. La aurora del pensamiento antropológico. Madrid: CSIC.

Caro Baroja, J. 1995. "Entrevista" de F. Esparza y J. Vizcay. Diario de Noticias, 03.09.95.

Chudnovsky, E. y J. Tejada 2011. El viaje de Cloe. Barcelona: Destino.

Council of Europe 2008. "Living together as equals in dignity". Estrasburgo, Ministerial Session.

Damasio, A. R. 1999. The feeling of what happens: Body and emotion in the making of consciousness. Nueva York: Harcourt Brace.

Damasio, A. R. 2010. Self comes to mind. Constructing the conscious brain. Nueva York: Pantheon Books.

DeGloma, Th. 2010. "Waking up in a contencious wordl" Hegehog Review 12 (2).

Deschamps, J. C. y T. Devos, 1996. "Relaciones entre identidad social e identidad personal”, en J. F Morales y otros (eds.), Identidad social. Valencia: Promolibro.

Díaz de Rada, A. 2010. Cultura, antropología y otras tonterias. Madrid: Trotta.

Díaz Viana, L. 2009. "La cultura como conquista: Naturaleza y condición en la definición de la identidad humana" Revista de Dialectología y Tradiciones Populares LXIV (1): $23-40$.

Disalvo, D. 2011. "Comunidades virtuales". Mente y Cerebro 48.

Douglas, M. 1983. "How identity problems disappear", en A. Jacobson-Widding (ed.), Identity: Personal and sociocultural. Upsala: Almqvist.

Dubar, C. 2000. La crise des identités. L'interprétation d'une mutation. París: PUF.

Duval, C. y otros autores 2009. "Le Soi à la loupe des neurosciences cognitives. De la conscience de soi à la conscience de l'autre”. Psychol NeuroPsychiatr Vieil 7 (1).

Elias, N. 1991. Die Gesellscahft der Individúen. Frankfurt am Main: Shurkampf.

Ellen, R. 2010. "Theories in anthropology and "anthropological theory". Journal of the Royal Anthropological Institute 16.

Ellis, G. F. R. 2009. "Top-down causation and the human brain", en N. Murphy y otros (eds.), Downward causation and the Neurobiology of free will. Berlín: Springer.

Escobar, M. y H. Román, 2010. "La presentación del yo en el ciberespacio. Un análisis de las autodefiniciones personales en blogs y redes sociales", en X Congreso de Sociología. Pamplona.

Farrugia, F. 2009. "Le syndrome narratif: théorie et terrain". Cabiers Internationaux de Sociologie 127. 
Fortes, M. 1987. Religion, morality and the person, Essays on Tallensi Religion. Cambridge: Cambridge University Press.

Geertz, C. 1973. The Interpretation of Culture. Nueva York: Basic Book.

Geertz, C. 2000. Available light: Anthropological reflections on philosophical topics. Princeton: Princeton University Press.

Georges, F. 2009. "Représentation de soi et identité numérique. Une approche sémiotique et quantitative de l'emprise culturelle du web 2.0". Réseaux 154 (2).

Gergen, K. J. 2009. Relational being: Beyond self and community. Oxford: Oxford University Press.

Goffman, E. 1959. The presentation of self in everyday life. Nueva York: Doubleday Anchor Books.

Goffman, E. 1972 [1963]. Stigma. Über Techniken der Bewältigung beschädigter Identität. Frankfurt am Main: Suhrkamp.

González García, J. M. 2001. "Metáforas de la subjetividad: Nosce te ipsum", en J. M. Iranzo y J. R. Blanco (eds.), Sobre identidades. Pamplona: Universidad Pública de Navarra.

Heritier, F. 1996. Masculin/Féminin. La pensée de la différence. París: Odile Jacob.

Honneth, A. 2010. Crítica del agravio moral. Patologias de la sociedad contemporánea. México: FCE.

Ingold, T. 1998. "Introduction to social life", en T. Ingold (ed.), Companion Encyclopaedia of Anthropology. Nueva York: Routledge.

Ingold, T. 2010. "Anthropology comes to life". General Anthropology 17 (1).

Juarrero, A. 2009. "Top-down causation and autonomy in complex systems", en N. Murphy y otros (eds.), Downward causation and the Neurobiology of free will. Berlín: Springer.

Kreiner, G. E. y otros autores 2006. "Where is the "me" among the "we"? Identity work and the search for optimal balance". Academy of Management Journal 49 (5).

Lenclud, G. 2008. "Identité et identities". L'Homme 187-188.

Lenclud, G. 2009. "Être une personne". Terrain 52.

León, M. de y M. F. A. Sanjuán, 2009. Las matemáticas y la física del caos. Madrid: CSIC.

Lévi-Strauss C. 1977. "Conclusion”, en J-M. Benoist y M. Serres (eds.), L'identité : séminaire interdisciplinaire 1974-1975. París: PUF.

Lévi-Strauss, C. 2008 [1955]. Tristes tropiques. Paris: Gallimard.

Lévy-Bruhl, L. 1927. L'âme primitive. París. F. Alcant.

Lisón Tolosana, C. 2010. Qué es ser hombre (valores cívicos y conflictivos en la Galicia profunda). Madrid: Akal.

Lizón, A. 2010. "Encrucijadas teóricas en la sociología del siglo xx". Papers 95 (2).

Luckman, Th. 1979. "Persönliche Identität, soziale Rolle und Rollendistanz", en O. Marquard y K. Stierle (eds.), Identität. Munich: Wilhelm Fink.

Lukes, S. 2000. "Different cultures, different rationalities?" History of the Human Sciences 13 (1).

Mauss, M. 1968. "Une catégorie de l'esprit humain: la notion de personne", en Sociologie et anthropologie. París: PUF.

MacKinnon, N. J. y D. R. Heise, 2010. Self, identity, and social institutions. Nueva York: MacMillan.

Mead, G. H. 2008 [1931]. Filosofía del presente. Madrid: CIS.

Morin, E. 2011. La voie. Pour l'avenir de l'humanité. París: Fayard.

Núñez Ladezéve, L. 2005. Identidades humanas: conflictos morales en la postmodernidad. Madrid: Centro de Estudios Políticos y Constitucionales.

Olmo, M. del 1994. "Una teoría para el análisis de la identidad cultural". Arbor 579. 
Owens, T. J. y otros autores 2010. "Three faces of identity". Annual Review of Sociology 36. Pérez Agote, A. 2006. "Identidad", en S. Giner y otros (eds.), Diccionario de Sociología. Madrid: Alianza.

Quante, M. 2007. "The social nature of personal identity" Journal of Consciousness Studies 14 (5-6).

Raulin, A. y D. Dussy, 2008. "A la rencontre de la personne" Cahiers Internationaux de Sociologie 124 (1).

Romagnoli, S. 2010. "Le corps propre: une pierre d'achoppement des théories contemporaines sur l'identité personnelle". Revue Philosophique de Louvain 108 (2).

Reza, G. A. de la 2010. Sistemas complejos. Barcelona: Anthropos.

Roesler, C. 2008. "The self in cyberspace. Identity formation in postmodern societies and Jung's self as an objective psyche" Journal of Analytical Psychology 53.

Sánchez de la Yncera, I. 2008. "Apostarse en presente (En la estela de G. H. Mead). Identidad y autotrascendencia en los ámbitos de interacción”, Introducción a Mead, G. H., Filosofía del presente. Madrid: CIS.

Schroll, M. 2010. "The Future of a Discipline: Considering the ontological/methodological future of the anthropology of consciousness, Part I". Anthropology of Consciousness 21 (1).

Sloterdijk, P. 2010. "Actio in distans. Sobre las formas de producción telerracional del mundo", en G. Aranzueque, (ed.), Ontología de la distancia. Madrid: Adaba.

Strathern, M. 2011. "Binary licence". Common Knowledge 17 (1).

Tomasello, M. 2010. ¿Por qué cooperamos?. Buenos Aires: Katz.

Tubau, D. 2011. El guión del siglo XXI. Madrid: Alba.

Wagner, R. 2001. An Anthropology of the subject. Los Angeles: University of California Press.

Wieviorka, M. 2011. "Entre el sujeto personal y global: ¿qué mediación?”, en D. Innerarity, y J. Solana, (eds.), La humanidad amenazada: gobernar los riesgos globales. Barcelona: Paidós.

Zubiri, X. 1986. Sobre el hombre. Madrid: Alianza.

Fecha de recepción: 31 de agosto de 2010

Fecha de aceptación: 5 de julio de 2011 